\title{
Resilienz des Grundgesetzes im Zeitalter des Populismus*
}

\begin{abstract}
Populists are gaining influence worldwide. It is evident from various examples around the globe that they can become a serious threat to liberal democracy by successively transforming democratic systems into autocracies. So far, hardly any effective means has been found to tackle the phenomenon. Reason enough to carefully assess the resilience of the Basic Law against authoritarian-populist attacks and to identify solutions for existing vulnarabilities.
\end{abstract}

\section{Résumé}

Les populistes gagnent en influence dans le monde entier. Il est évident, d'après divers exemples dans le monde, qu'ils peuvent devenir une menace sérieuse pour la démocratie libérale en transformant successivement les systèmes démocratiques en autocraties. Jusqu'à présent, aucun moyen efficace pour faire face à ce phénomène n'a réellement été trouvé. Il s'agit là d'une raison suffisante pour évaluer soigneusement la résistance de la loi fondamentale aux diverses attaques populistes et autoritaires ainsi que pour identifier des solutions aux vulnérabilités existantes.

Populistische Parteien gewinnen weltweit an Einfluss. Verschiedene Beispiele rund um den Globus zeigen, dass Populisten zu einer ernsthaften Bedrohung für die liberale Demokratie werden können, wenn sie demokratische Systeme - unter Berufung auf den „wahren Willen des Volkes“ - sukzessive zu Autokratien umformen. Bislang fehlt es an wirksamen Maßnahmen, um dem Phänomen Herr zu werden. Grund genug die Resilienz des Grundgesetzes gegen autoritär-populistische Übergriffe einer kritischen Überprüfung zu unterziehen und erste Lösungsansätze für erkannte Schwächen in die Diskussion einzubringen.

Möchte man die verfassungsrechtlichen Implikationen des global erstarkenden Populismus analysieren, bedarf es zunächst einer terminologischen Annäherung (I.). Bei dem Begriff „Populismus“ handelt es sich um eine sozialwissenschaftliche Kategorie, die viele unterschiedliche politische Phänomene erfasst. Die Ursachen für den wachsenden Erfolg dieser Politikform sind komplex und kontextabhängig - einfache Lö-

* Anika Klafki ist Juniorprofessorin (tenure track) für Öffentliches Recht (insbes. transnationales Verwaltungsrecht) an der Friedrich-Schiller-Universität Jena und Habilitandin von Prof. Dr. Hermann Pünder, LL.M. (Iowa) an der Bucerius Law School. Der Text beruht auf einem Vortrag, den die Verfasserin im Rahmen des 5. Biannual German-South African Dialogue on Democracy zum Thema „Democracy in Times of Populism” am 7. Februar 2020 an der Universität Stellenbosch (Südafrika) gehalten hat. Die Verfasserin dankt Jelena Bäumer (Universität Lüneburg), Henk Botha (Stellenbosh University), Wessel le Roux (University of the Western Cape), Nils Schaks (Universität Mannheim) und Dominik Steiger (Technische Universität Dresden) für die Organisation und allen Beteiligten für die anregende Diskussion. 
sungen gibt es nicht. Beschränkt man den Untersuchungsgegenstand auf den sog. autoritären Populismus so lassen sich Widersprüche populistischer Grundannahmen mit der liberalen Demokratie des Grundgesetzes feststellen (II.). Gleichwohl erweisen sich die Waffen der ,wehrhaften Demokratie“ im Umgang mit autoritären Populisten als stumpf. Betrachtet man die Rechtsentwicklungen in Polen, Ungarn und der Türkei, zeigt sich, dass autoritäre Populisten, die eine Regierungsmehrheit erreichen, wirkmächtige Vetospieler - allen voran die Justiz - angreifen, um das demokratische Staatswesen sukzessive zu untergraben (III.). Auch das deutsche Grundgesetz ist vor solchen Gefahren nicht gefeit. Die Resilienz der Verfassung gegen autoritäre Übergriffe ließe sich durch moderate Verfassungsergänzungen zum Schutze der Unabhängigkeit des Bundesverfassungsgerichts beträchtlich erhöhen (IV.).

\section{A. Populismus - Eine begriffliche Annäherung}

Kurz nach der Wahl von Trump zum Präsidenten der Vereinigten Staaten erkannte das Cambridge Dictionary 2017 den Begriff „Populismus“ als Wort des Jahres an. ${ }^{1}$ Dem Wörterbuch zufolge beschreibt Populismus ,,politische Ideen und Aktivitäten, die darauf abzielen, die Unterstützung der einfachen Leute zu erhalten, indem man ihnen gibt, was sie wollen“. Der Begriff hat eine negative Konnotation; er drückt üblicher Weise Missbilligung aus. Eine Herausforderung für die rechtswissenschaftliche Beschäftigung mit dem Phänomen besteht darin, dass dem Terminus angesichts seiner weiten deskriptiven Natur eine gewisse Beliebigkeit innewohnt. Gebraucht man den Populismusbegriff als abwertenden politischen Kampfbegriff, so büßt er seine wissenschaftliche Operabilität ein. ${ }^{2}$

Trotz der begrifflichen Unschärfe werden namenhafte Politikwissenschaftlerinnen und -wissenschaftler - wie etwa Mounk, Norris und Inglehart - nicht müde, zu betonen, dass der Populismus eine ernsthafte Bedrohung für die liberale Demokratie darstellt. ${ }^{3}$ In der Politikwissenschaft wurden mehrere Definitionen des Populismus formuliert. Das Verständnis des Phänomens ist jedoch bei weitem nicht einheitlich. Während einige den Populismus als eine Ideologie betrachten, ${ }^{4}$ beschreiben andere ihn als ein bloßes rhetorisches Stilmittel. ${ }^{5}$

\section{Beschränkung auf den autoritären Populismus}

Es gibt zwei Merkmale, die dem Populismus gemeinhin zugeschrieben werden: Erstens sind Populisten elitenkritisch und stellen die Legitimität des „Establishments“ in

1 Siehe https://dictionaryblog.cambridge.org/2017/11/29/cambridge-dictionarys-word-of-theyear-2017/ <07/2020>.

2 Krit. daher zur liberalen Populismuskritik Marchart, APuZ 67 (44-45/2017), 10 (12 ff9.).

3 Mounk, The People vs. Democracy, 2018, S. 3; Norris/Inglehart, Cultural Backlash, 2019, S. 409 ff.; Zürn, Autoritärer Populismus vs. offene Gesellschaft - eine neue Konfliktlinie, 2018, S. 4.

4 Mudde/Kaltwasser, Populism, 2017, S. 6.

5 Norris/Inglehart (Fn. 3), S. 4. 
Frage. Zweitens sehen Populisten den „wahren Wille des Volkes“ als einzige legitime Quelle staatlicher Autorität an. ${ }^{6}$ Die Begriffe ,,Volk“, „Elite“ und ,,allgemeiner Wille“ sind somit Schlüsselbegriffe des Populismus. Beschränkt man sich auf diese Definitionsmerkmale so stellt sich Populismus als bloßes Konzept oder politisches Verhaltensmuster dar, ${ }^{7}$ das leicht mit umfassenderen politischen Weltanschauungen wie (Neo-)Liberalismus, Sozialismus oder Faschismus kombiniert werden kann.

Aus verfassungsrechtlicher Perspektive sind vor allem diejenigen populistischen Strömungen kritisch zu betrachten, die einen anti-pluralistischen Volksbegriff vertreten und darauf aufbauend eine ausschließende Form der Identitätspolitik betreiben. ${ }^{8}$ Die folgende Untersuchung soll auf diese Form des Populismus, die auch als ,autoritärer Populismus“ bezeichnet wird, ${ }^{9}$ beschränkt werden. Ein Markenzeichen autoritärer Populisten ist es, dass sie den „wahren Willen des Volkes“ strikt majoritär konstruieren, und alle vom Volksbegriff ausschließen, die vom vermeintlichen Mehrheitswillen abweichen. Auf diese Weise wird die Gesellschaft in zwei homogene, antagonistische Lager gespalten: „das wahre Volk“ und „die korrupte Elite“. ${ }^{10}$ Andersdenkende werden als „Feinde des Volkes“ oder „Volksverräter" diffamiert. ${ }^{11}$ Ihnen werden unter Berufung auf den legitimen Mehrheitswillen Freiheiten und Teilhaberechte abgesprochen. Der autoritäre Populismus hat insoweit zugleich eine anti-liberale wie anti-egalitäre Prägung. Ferner bedingt die strikte Bindung an den Willen des Nationalvolkes eine anti-multilaterale Ausrichtung, die Einflussnahmen internationaler Akteure auf das politische System - sofern sie nicht dem vermeintlichen Mehrheitswillen entsprechen - delegitimiert. ${ }^{12}$

Der Begriff des autoritären Populismus umfasst nicht nur Rechtspopulisten, die derzeit in Europa an Einfluss gewinnen. Auch Linkspopulisten, die in der südamerikanischen Politik eine wichtige Rolle spielen, können als autoritäre Populisten bezeichnet werden, sofern sie eine ausschließende Form der Identitätspolitik propagieren. Während der autoritäre Rechtspopulismus die Grenze zwischen „dem Volk“ und den illegitimen „Anderen“ auf Grundlage nationalistischer, ethnischer oder religiöser Merkmale zieht, rekurriert der Linkspopulismus auf sozial-ökonomische Klassenzugehörigkeiten. ${ }^{13}$ Der autoritäre Populismus stellt folglich keine eigenständige Weltanschauung dar, sondern ist ein Vehikel für substanzielle politische Ideologien. ${ }^{14}$

6 Norris/Inglehart (Fn. 3), S. 4 ff.

7 Tushnet, German Law Journal 2019, 382 (382).

8 Siehe dazu auch Kersten, JuS 2018, 929 ff.

9 Wie hier Möller, ZfP 2019, 430 (437f.). Auch hinsichtlich des „autoritären Populismus“ gibt es unterschiedliche Definitionen. Siehe etwa die Begriffsbestimmungen bei Norris/Inglehart (Fn. 3), S. 4 ff. und Zürn (Fn. 3), S. 3 ff. Eine ähnliche Begriffsbeschränkung wie hier hält auch Voßkuhle, Der Staat 2018, 119 (121 ff.) für eine rechtswissenschaftliche Befassung für notwendig.

10 Mudde/Kaltwasser (Fn. 4), S. 6, 9 ff.

11 Müller, What Is Populism?, 2016, S. 3 ff., 20; Möller, ZfP 2019, 430 (437).

12 Zürn (Fn. 3), S. 3, 7 ff.

13 Tushnet, German Law Journal 2019, 382 (388 f.); Arato, Populism, Constitutional Courts, and Civil Society, in: Landfried (Hrsg.), Judicial Power, S. 318 (327).

14 Tushnet, German Law Journal 2019, 382 (389). 


\section{Ursachen für das Gedeihen des autoritären Populismus und ver- meintlich einfache Lösungen}

Populistische Mobilisierung erweist sich in solchen Gesellschaften als besonders effektiv, in denen sich die Wählenden von den etablierten Parteien entfremdet und von den Regierenden nicht angemessen repräsentiert fühlen. ${ }^{15}$ Gilens und Page haben kürzlich gezeigt, wie gerechtfertigt diese Annahme für das US-System ist. Ihre empirischen Studien zeigen, dass wirtschaftliche Eliten und Interessengruppen erheblichen Einfluss auf die Politik nehmen können, während Durchschnittsbürger und nicht-wirtschaftliche Verbände kaum Einfluss auf politische Entscheidungen haben. ${ }^{16}$ Auch in Deutschland erweisen sich einkommensstarke Bevölkerungsschichten in empirischen Studien als einflussreicher als einkommensschwache. ${ }^{17}$ Eine Strategie, um die Responsivität der liberalen Demokratie zu erhöhen, ist daher die Ausweitung und Verbesserung staatlicher Partizipationsmechanismen. ${ }^{18}$ Den höchsten Grad an Entscheidungsbeteiligung vermitteln direkt-demokratische Verfahren. ${ }^{19}$ In Demokratien, die keine Tradition plebiszitärer Formen der Entscheidungsfindung haben, besteht jedoch die große Gefahr, dass Populisten neue direktdemokratische Entscheidungsmechanismen missbrauchen und instrumentalisieren. Das Brexit-Referendum in Großbritannien ist ein anschauliches Beispiel dafür. In Systemen, in denen Populisten bereits auf dem Vormarsch sind, ist eine Änderung der Verfassungsordnung hin zu einer direkteren Demokratie daher riskant. ${ }^{20}$

Statt systemischer Verfassungsänderungen sind politische Lösungen gefragt, die die Parteipolitik wieder stärker an den Präferenzen der Bevölkerung ausrichten. Der Politikwissenschaftler Mounk weist indes zu Recht darauf hin, dass auch dieser Weg für liberale Demokratien steinig ist; denn empirische Studien zeigen, dass illiberale Überzeugungen in der Bevölkerung westlicher Staaten an Zuspruch gewinnen. ${ }^{21}$ Über die Ursachen dieser Entwicklung gibt es keine zuverlässigen Informationen. Während soziale Spaltungen und ungleiche Wohlstandsverteilung oft im Verdacht stehen, illiberale Präferenzen zu begünstigen, ${ }^{22}$ spiegelt sich das in der Wählerschaft populistischer Parteien nicht wider. ${ }^{23}$ So sind die Wähler populistischer Parteien in Europa nicht nur in sozio-ökonomisch schwachen Bevölkerungsschichten zu finden. Es handelt sich

15 Roberts, Populism, Political Mobilizations, and Crises of Political Representation, in: La Torre, The Promise and Perils of Populism, S. 140 (147 ff.); Alterio, Global Constitutionalism 2019, 270 (270 ff.); Hawkins/Read/Pauwels, Populism and Its Causes, in: Kaltwasser/ Taggart/Ochoa/Ostiguy (Hrsg.), The Oxford Handbook of Populism, S. 267 (279 f.).

16 Gilens/Page, Perspectives on Politics 2014, $564 \mathrm{ff}$.

17 Elsässer/Hense/Schäfer, ZPol 27 (2017), 161 ff. Speziell zur Einhegung des Lobbyismus Pünder, VVDStRL 72 (2013), 191 (237 ff.).

18 Alterio, Global Constitutionalism 2019, 270 (270 ff.).

$19 \mathrm{Zu}$ den Grenzen dieses Entscheidungsmechanismus prägnant Pünder, VVDStRL 72 (2013), 191 (196).

20 Kritisch auch Voßkuhle, Der Staat 2018, 119 (128 f.). Für die Öffnung der Bundesgesetzgebung für Formen der direkten Demokratie hingegen Kersten, JuS 2018, 929 (936).

21 Mounk (Fn. 3), S. 13.

22 So etwa Tushnet, German Law Journal 2019, 382 (384 f.).

23 Müller (Fn. 11), S. 14. 
vielmehr um ein klassenübergreifendes Phänomen. ${ }^{24}$ Die Alternative für Deutschland wurde beispielsweise lange überwiegend von Personen mit überdurchschnittlichem Einkommen gewählt. ${ }^{25}$ Auch spricht die Tatsache, dass Populisten - etwa in Polen und der Türkei - gerade in Phasen wirtschaftlicher Prosperität starke Zuwächse erlebten, gegen ein rein ökonomisches Erklärungsmodell. ${ }^{26}$ Politische Antworten auf die Bedrohung der liberalen Demokratie durch Populisten zu finden, ist angesichts der komplexen Ursachen ein schwieriges Unterfangen. ${ }^{27}$ Obwohl die Corona-Pandemie das Mobilisierungspotenzial von Populisten aktuell schwächt, ${ }^{28}$ ist ein Ende des Höhenflugs populistischer Akteure noch lange nicht in Sicht.

\section{B. Autoritärer Populismus und Verfassungsrecht}

Die autoritär-populistische Konstruktion der Repräsentation, die Idee der Homogenität des Volkes und die daraus resultierende Diskreditierung von Minderheitenpositionen konfligieren mit der liberalen Demokratie des Grundgesetzes (1.). Gleichwohl erweisen sich die Verteidigungsmechanismen der wehrhaften Demokratie als ungeeignet, um die von Populisten ausgehende Bedrohung abzuwehren (2.).

\section{Konflikt verfassungsrechtlicher und populistischer Grundannah- men}

Das Grundgesetz bestimmt in Art. 20 Abs. 2, dass alle Staatsgewalt vom Volke ausgeht. Dem würden auch autoritäre Populisten zustimmen. Viele populistische Bewegungen machen sich explizit für direkt-demokratische Entscheidungsmechanismen stark, um die Politik enger an den Mehrheitswillen zu binden. ${ }^{29}$ Auch Art. 38 Abs. 1 S. 2 GG kann in einer Weise gelesen werden, die dem populistischen Repräsentationsideal entspricht. Danach sind die Bundestagsabgeordneten Vertreter des ganzen Volkes. Populisten kritisieren oft, dass die Abgeordneten nicht den Willen des „,wahren Volkes“" vertreten, sondern die Interessen einer illegitimen Elite. ${ }^{30}$ Man könnte daher

24 Vgl. zur Entwicklung in Polen Matthes, Problems of Post-Communism 2016, 288 (293 ff.).

25 Siehe Niedermayer/Hofrichter, ZParl 2016, 267 (270 ff.).

26 Zürn (Fn. 3), S. 9.

27 Ausf. zu den gegenwärtigen Reaktionsmustern Möller, ZfP 2019, 430 (441ff.).

28 Ausweislich der Wahlumfragen von infratest dimap, hat die AfD während der Corona-Pandemie ein Drittel ihrer potenziellen Wähler eingebüßt, https://www.wahlrecht.de/umfragen/ dimap.htm $<07 / 2020>$.

29 Mounk(Fn. 3), S. 49 f.

30 Siehe etwa die Aussagen von Björn Höcke (Landesvorsitzender der AfD Thüringen) "Was die gesamte bürgerliche Opposition eint, ist die grundsätzliche Ablehnung der lebensfeindlichen Ideologie, mit der die Herrschenden ihre Politik legitimieren.”, Compact-Konferenz, 27.11.2017 https://www.bjoern-hoecke.de/single-post/2017/11/27/Bj\%C3\%B6rn-H\%C3\% B6cke-auf-der-Compact-Konferenz $<07 / 2020>$; "Wer das Treiben der uns leider noch beherrschenden Politiker beobachtet, der kann kaum den Eindruck gewinnen, dass hier moralisch integere Menschen am Werke sind." (Min. 12) "[...] die deformierte politisch Klas- 
meinen, dass Populisten sich zwar einer aggressiven Rhetorik bedienen, im Übrigen aber die demokratische Idee des Grundgesetzes teilen. ${ }^{31}$

In Art. 21 Abs. 1 GG heißt es jedoch: „Die Parteien wirken bei der politischen Willensbildung des Volkes mit.” Die ausschließliche Verwendung des Plurals „Parteien“ in Art. 21 GG verdeutlicht, dass die Verfassung dem Parteienpluralismus verpflichtet ist und sich bewusst vom Einparteiensystem der nationalsozialistischen Diktatur abgewendet hat. ${ }^{32}$ Die Verfassung basiert folglich nicht auf einer homogenen Konzeption des Willens des Volkes. Vielmehr erkennt das Grundgesetz an, dass die Gesellschaft aus einer Vielzahl von fluiden, sich überlappenden Interessengruppen besteht. ${ }^{33}$

Dieses Volksverständnis spiegelt sich auch im vom europäischen Recht geprägten deutschen Verwaltungsrecht wider. Dort finden sich sektorspezifisch ausdifferenzierte Beteiligungsverfahren, die in unterschiedlichem Maße Entscheidungsmacht in die Hände von Bürgern und Betroffenen legen. Insbesondere das städtebauliche und infrastrukturbezogene Planungsrecht normiert breite Öffentlichkeitsbeteiligungen, um sicherzustellen, dass alle in der Gesellschaft vertretenen Interessen im administrativen Entscheidungsprozess berücksichtigt werden können. ${ }^{34}$ Das deutsche Sozialrecht verfügt ferner über korporatistische Arrangements, in denen staatliche Entscheidungen unmittelbar in die Hände privater Interessengruppen gelegt werden. So wird beispielsweise das Leistungsrecht der gesetzlichen Krankenversicherung in Deutschland weitgehend von einem Gremium geregelt, in dem Vertreter von Ärzten und Krankenhäusern einerseits und Vertreter der Krankenkassen andererseits den Katalog der medizinischen Leistungen aushandeln. ${ }^{35}$ Die populistische Vorstellung eines homogenen Volkswillens steht also nicht nur im Widerspruch zur verfassungsrechtlich garantierten Parteiendemokratie, sondern widerspricht auch dem pluralen Gesellschaftsbild, von dem das gesamte deutsche und europäische Öffentliche Recht geprägt ist. ${ }^{36}$

Noch deutlicher wird die vom autoritären Populismus ausgehende Bedrohung verfassungsrechtlicher Kernprinzipien, wenn man seine anti-liberalen und anti-egalitären Züge in den Blick nimmt. Teilen der Gesellschaft das Recht zur politischen Teilhabe entweder aus ethno-nationalistischen Gründen oder durch soziale Klassenkonstruktionen - abzusprechen, steht im Widerspruch zur verfassungsrechtlich in Art. 3 GG verbürgten Gleichheit und den politischen Freiheitsgrundrechten. Demokratische Verfassungen suchen seit jeher eine „Tyrannei der Massen“ zu Lasten von Minderheiten zu verhindern. ${ }^{37}$ Freilich unterscheidet auch das Verfassungsrecht bei der Konstruktion des Wahlvolkes zwischen Deutschen, Unionsbürgern und sogenannten Drittstaatsan-

se [...]" (Min. 16), Rede bei Pegida, 14.5.2018, https://www.youtube.com/watch?time_cont inue $=780 \& \mathrm{v}=\mathrm{hp} 6 \mathrm{yOrVUbs} 8 \&$ feature $=\mathrm{emb} \log _{\mathrm{o}}<07 / 2020>$.

31 Mounk (Fn. 3), S. 52 begreift Populisten daher als illiberale Demokraten. Auch Moffitt, The Global Rise of Populism, 2016, S. 142 f. erkennt die demokratische Grundtendenz des Populismus an.

32 Siehe statt vieler Klafki, in: Münch/Kunig, Grundgesetz, 7. Aufl. 2021 (i.E.), Art. 21 Rn. 2.

33 Allgemein dazu Morlok, in: Dreier, Grundgesetz, 2015, Art. 21 Rn. 1. Im Populismus-Kontext siehe Voßkuhle, Der Staat 2018, 119 (122 ff.); Kersten, JuS 2018, 929 (930); Martins, RuP 2018, 286 (291 ff.).

34 Klafki, Der Staat 2019, 367 (369 ff.).

35 Klafki/Loer, VerwArch 2017, 343 (343 ff.).

36 Voßkuhle, Der Staat 2018, 119 (122 ff.).

37 Siehe dazu schon Mill, Liberty, 1913, S. 3. 
gehörigen. Abgesehen davon sind Diskriminierungen aufgrund von Rasse, Heimat und Herkunft - wie im Rechtspopulismus gefordert - ebenso wie Benachteiligungen wegen weltanschaulicher Positionen - wie sie im linkspopulistischen Spektrum vorkommen - gem. Art. 3 Abs. 3 GG unzulässig.

Werden Minderheiten- und Oppositionsrechte eingeschränkt, so beschädigt dies unmittelbar die Funktionsbedingungen der Wettbewerbsdemokratie. ${ }^{38}$ Je weniger Raum für die Artikulation anderer Ansichten vorhanden ist, desto weniger offen ist das politische System für demokratische Machtwechsel. Sog. ,illiberale Demokratien“, in denen die demokratisch gewählten Regierungs- bzw. Regierungsfraktionsvertreter politische Freiheiten Oppositioneller einschränken oder aussetzen, sind daher, bei Lichte betrachtet, keine demokratischen Systeme mehr. Vielmehr handelt es sich - angesichts der verzerrten Wettbewerbsbedingungen - um verdeckt autoritäre Systeme, in denen Wahlen ihre legitimatorische Wirkung nicht mehr wirksam entfalten können. ${ }^{39}$

\section{Schwäche verfassungsrechtlicher Schutzmaßnahmen gegenüber populistischen Akteuren}

Trotz dieser Friktionen des autoritären Populismus mit dem Demokratieprinzip lässt sich der Aufstieg autoritär-populistischer Akteure - wie sich in Polen und Ungarn nachzeichnen lässt - mit den Mitteln des Verfassungsrechts kaum stoppen.

\section{Neutralität als Kernprinzip der Wettbewerbsdemokratie}

So ist es einer freiheitlich-demokratischen Verfassungsordnung unmöglich, die deskriptiv-analytische Definition des autoritären Populismus zu einer rechtlichen Kategorie umzuformen und konkrete Rechtsfolgen daran zu knüpfen. Freiheitlich-demokratische Verfassungen sind auf die Neutralität des Rechts und staatlicher Organe gegenüber politischen Äußerungen und Parteibewegungen angewiesen. ${ }^{40}$ Sinn der Verfassung ist es nicht, richtige politische Ansichten von falschen zu unterscheiden, sondern einen fairen politischen Wettbewerb um die Stimmen der Wählerschaft zu ermöglichen. Das innovative Potenzial der Demokratie resultiert aus einer permanenten Spannung zwischen Regierung und Opposition. ${ }^{41}$ Im politischen Meinungskampf sind überspitzte politische Äußerungen üblich und können nur in extremen Fällen Einschränkungen der Meinungsfreiheit rechtfertigen. ${ }^{42}$ Die verfassungsrechtliche Neutralitätsverpflichtung hält das politische System offen für demokratische Machtwech-

38 Grundlegend zur Wettbewerbsdemokratie Grimm, in: Benda et al (Hrsg.), Handbuch des Verfassungsrechts, 2. Aufl. 1994, § 14 Rn. 6 ff., 42 ff.; Kotzur, VVDStRL 69 (2010), 173 (179 ff.); Hatje, VVDStRL 69 (2010), 135 (143 ff.).

39 Voßkuhle, Der Staat 2018, 119 (130 f.).

40 Ausf. hierzu Kuch, AöR 142 (2017), 491 (508 ff.). A.A. im Hinblick auf Äußerungsbefugnisse von Amtsinhabern Payandeh, Der Staat 55 (2016), 519 (535 ff.).

41 St. Augsberg, in: Heinig/Terhechte (Hrsg.), Postnationale Demokratie, 2013, S. 27 (43 ff.).

42 Burkhardt/Peifer, in: Wenzel (Hrsg.), Das Recht der Wort- und Bildberichterstattung, 6. Aufl. 2018, 10. Kap. Rn. 64 ff. 
sel. ${ }^{43}$ Würde in einem Staatssystem eine bestimmte politische Meinung rechtlich bevorzugt, könnte man kaum mehr von einer Demokratie sprechen. ${ }^{44}$

Darüber hinaus können anti-elitäre politische Äußerungen - die ein Erkennungsmerkmal von Populisten sind - durch die tatsächlichen Umstände gerechtfertigt sein. In Ungarn oder Italien beispielsweise kritisierten populistische Parteien ursprünglich Korruptionsskandale von Regierungsparteien. ${ }^{45} \mathrm{Zu}$ Recht wurden hier also bestehende Ungerechtigkeiten angeprangert. In der Phase der Entstehung populistischer Bewegungen ist das Recht daher weitgehend blind für die daraus erwachsenden Gefahren.

\section{Unwirksamkeit von Parteiverboten und Finanzierungsausschlüssen}

Gelegentlich wird die Forderung laut, die von Art. 21 Abs. 2 und 3 GG bereitgehaltenen Waffen der „wehrhaften Demokratie“ gegen autoritäre Populisten einzusetzen. ${ }^{46}$ Sowohl rechtlich als auch faktisch stößt man dabei jedoch auf Hindernisse.

\section{a) Populisten als Feinde der freiheitlich-demokratischen Grundordnung?}

Nach Art. 21 Abs. 2 GG ,,sind Parteien, die nach ihren Zielen oder nach dem Verhalten ihrer Anhänger darauf ausgehen, die freiheitliche demokratische Grundordnung zu beeinträchtigen oder zu beseitigen" verfassungswidrig, sofern das BVerfG - auf Antrag des Bundestages, des Bundesrates oder der Bundesregierung ${ }^{47}$ - diese Feststellung trifft. Dass autoritäre Populisten Feinde der freiheitlich-demokratischen Grundordnung sind, ist im Parteiverbotsverfahren nicht leicht zu belegen. Sowohl materiellals auch verfahrensrechtlich stellt das BVerfG bekanntlich höchste Anforderungen. Die freiheitlich-demokratische Grundordnung umfasst nach dem jüngsten NPD-Verbotsurteil drei Kernelemente: die Menschenwürde, das Demokratie- und das Rechtsstaatsprinzip. ${ }^{48}$ Das Prinzip der Demokratie garantiert die gleichberechtigte Beteiligung aller Gesellschaftsmitglieder am Prozess der politischen Entscheidungsfindung und die Rückbindung der Ausübung der Staatsgewalt an das Volk. Zum Demokratieprinzip gehören auch das Mehrparteienprinzip, die Chancengleichheit für politische Parteien, der Parlamentarismus und die Forderung nach periodischen freien Wahlen. Die Garantie der Menschenwürde sichert darüber hinaus die demokratischen Grundrechte und das Prinzip der bürgerlichen Gleichheit ab. Man könnte daher meinen, dass das Verbot populistischer Parteien nicht allzu schwierig sein sollte, da - wie bereits gesehen - die Grundannahmen autoritärer Populisten gegen diese Werte verstoßen.

43 Larmore, Patterns of moral complexity, 1987, S. $42 \mathrm{ff}$. Kritisch aber Mouffe, Ratio Juris 1994, $314 \mathrm{ff.}$.

44 Rawls, Political Liberalism, 1993, S. 10.

45 Vgl. Mudde/Kaltwasser, Populism, 2017, S. 100. Siehe zur Situation in Ungarn Lendvai, Foreign Affairs 98 (2019), 44 (50).

46 Siehe etwa die Forderung von Prantl, in Süddeutsche v. 27.7.2019, https://www.sueddeutsc he.de/politik/rechtsextremismus-die-afd-driftet-nach-rechts-draussen-1.4540706 $<07 / 2020>$.

$47 \S 43$ Abs. 1 BVerfGG.

48 BVerfGE 144, 20 (205 ff.). 
Es gelten jedoch sehr hohe Anforderungen für den Beleg der antidemokratischen Haltung einer Partei. ${ }^{49}$ Der ständige Bezug von Populisten auf den Willen des Volkes macht es schwer, ihnen antidemokratische Überzeugungen nachzuweisen. ${ }^{50}$ Die Politikwissenschaftlerin Mouffe bewertet den Populismus sogar als eine legitime diskursive Strategie, um eine gerechtere ,radikale Demokratie“ zu verwirklichen. ${ }^{51}$ Auch ist bei neu gegründeten populistischen Bewegungen oft unklar, wie sie den ,wahren Volkswillen“ umsetzen wollen. Selbst wenn Populisten den anti-pluralistischen Anspruch formulieren, dass sie allein den Volkswillen richtig interpretieren, rechtfertigt diese Aussage allein kaum rechtliche Eingriffe. Solche Behauptungen können als schlichte Wahlwerbung angesehen werden. Zudem formulieren Rechtspopulisten rassistische Äußerungen oft so, dass sich daraus keine konkreten politischen Forderungen ableiten lassen und Raum für Interpretation bleibt. ${ }^{52}$ Der Inhalt der Äußerungen populistischer Parteieliten hält sich daher vielfach noch im Rahmen des rechtlich Zulässigen.

Selbst bei explizit geplanten Verfassungsänderungen ist es schwierig, sie als Verletzung des Demokratieprinzips zu brandmarken. Betrachtet man die Verfassungsänderungen, die bisher unter populistischen Führern wie Orbán, Erdoğan oder Kaczyński und Duda vorgenommen wurden, ist es kaum möglich, den genauen Wendepunkt zu markieren, an dem sich der demokratische Staat zu einem autoritären wandelt. ${ }^{53}$ Viele der von autoritären Populisten herbeigeführten Verfassungsänderungen finden ihre Entsprechung in anderen demokratischen Verfassungssystemen. Angesichts des komplexen Zusammenspiels demokratischer Institutionen in der Verfassungsrealität lassen sich politische Systemumbrüche nicht aus einzelnen Gesetzes- oder Verfassungsänderungen ablesen. Das Recht ist gegenüber Veränderungen der politischen Macht zumindest halbblind, da Normen staatliche Ämter abstrakt konstruieren - also ohne Rücksicht auf die Parteizugehörigkeit und die persönliche Ausrichtung der jeweiligen Amtsinhaber, die jedoch in der Verfassungsrealität entscheidend ist, um Abhängigkeiten und Machtnetzwerke erkennen zu können. ${ }^{54}$

Um den Missbrauch der ,schärfste(n) und überdies zweischneidige(n) Waffe des demokratischen Rechtsstaats" zu verhindern, hat das BVerfG zudem jüngst klargestellt, dass ein Parteienverbot nur in Betracht kommt, wenn die Möglichkeit besteht, dass eine Partei ihre verfassungsfeindlichen Ziele tatsächlich in die Realität umsetzen kann (Potenzialität). ${ }^{55}$ Damit nähert sich das Gericht der strengen Rechtsprechung des EGMR an, der insbesondere zahlreiche Parteienverbote in der Türkei als konventions-

49 Siehe BVerfGE 107, 339 (367 ff.); E 144, 20 (166 ff.).

50 Mounk (Fn. 3), S. 46 ff. begreift Populisten daher als illiberale Demokraten.

51 Mouffe, For a Left Populism, 2018, S. 5.

52 Siehe dazu exemplarisch die Entscheidung des VG Berlin, 7. 9.2011, Az.: 1 L 293.11, juris zu den NPD-Wahlplakaten mit der Aufschrift „Gas geben“.

53 Siehe zu dem Problem Tushnet, German Law Journal 2019, 382 (386).

54 Für eine stärkere Einbeziehung machtpolitischer Zusammenhänge in das Parteienrecht Schönberger JZ 2017, $701 \mathrm{ff}$.

55 BVerfGE 144, 20 (224 ff.). Für eine entsprechende Einschränkung schon Maurer, AöR 96 (1971), 203 (229 f.) (,potentielle Gefährlichkeit“). Krit. zum Erfordernis der Potenzialität Hillgruber, JA 2017, 398 (400); Kloepfer, NVwZ 2017, 913; Kluth, ZParl 2017, 676 (686); Linke, DÖV 2017, 483 (490). 
widrig verurteilt hat. ${ }^{56}$ Einerseits schwächt diese enge Auslegung von Art. 21 Abs. 2 GG die wehrhafte Demokratie, da sich autoritär-populistische Bewegungen nicht im Keim ersticken lassen. Andererseits erhöht die Betonung des Ausnahmecharakters des Parteiverbots zugleich die Resilienz unseres Verfassungssystems für den Fall, dass autoritäre Populisten eine Regierungsmehrheit erreichen und versuchen, sich unliebsamer Konkurrenten mittels Parteiverboten zu entledigen. Inwiefern der Finanzierungsausschluss für verfassungswidrige Parteien, die das Potenzialitätskriterium nicht erfüllen, eine gelungene Verfassungsinnovation darstellt, wird sich noch zeigen. ${ }^{57}$

\section{b) Politische Hindernisse für Parteiverbotsverfahren gegen populistische Partei- en in der Verfassungsrealität}

Auch wenn eine autoritär-populistische Bewegung die Schwelle der Potenzialität nachweislich überschritten hat, ist es eine heikle politische Entscheidung, ein Parteiverbots- oder Finanzierungsausschlussverfahren gegen eine wirkmächtige Oppositionspartei einzuleiten. Zu Recht wird immer wieder betont, dass ein Verbotsverfahren gegen Parteien, die sich zu einem ernsthaften politischen Gegner der Regierungsparteien entwickelt haben, aus Gründen der Verfassungsklugheit nicht mehr in Betracht kommt. ${ }^{58}$ So könnte ein Parteiverbotsverfahren in diesem Stadium als ein unrechtmäßiger Versuch der regierenden „Eliten“ erscheinen, ihre Macht zu erhalten und die Position der Populisten weiter stärken. Die gängige Narration populistischer Bewegungen, das Establishment versuche, sie mundtot zu machen, würde dadurch bestätigt und die gesellschaftliche Spaltung weiter vertieft. .59

So erklärt es sich auch, dass es in der Verfassungsrealität Europas bislang kaum Bemühungen gab, populistische Parteien zu verbieten. Immerhin konnte man in Deutschland zunächst einen kleinen Erfolg erzielen. So führte die Beobachtung des AfD-Parteiflügels der Flügel durch den Verfassungsschutz dazu, dass er aufgelöst wurde. ${ }^{60}$ Allerdings offenbart schon die Begrenzung der Beobachtung auf den Flügel eine gewisse Hilflosigkeit im Umgang mit der AfD; denn der Flügel war weder rechtlich noch organisatorisch unabhängig von der AfD. Der „Ehrenvorsitzende“61 der Par-

56 Siehe zur Rezeption der EGMR-Rspr. BVerfG 144, 20 (234 ff.). Siehe auch EGMR (The United Communist Parteies and others/Türkei), 30.1.1998 - 19392/92, ECHR 1998-I, 2 ff.; EGMR (Socialist Party and others/Türkei), 25.5.1998 - 21237/93, ECHR 1998-III, 1234 ff.; EGMR (ÖZDEP/Türkei), 8.12.1999 - 23885/94, ECHR 1999-VIII, 295 ff.; EGMR (Yazar and others/Türkei), 9.4.2002 - 22723/93 u.a., ECHR 2002-II, 397 ff.; EGMR (Sendak and others/Türkei, Nr. 2), 11.6.2002 - 25144/94, ECHR 2002-IV, 295 ff.; EGMR (Refah Partisi and others/Türkei), 13.2.2003 - 41340/98 u.a., ECHR 2003-II, $296 \mathrm{ff}$.

57 Krit. dazu Kingreen, Jura 2017, 499 (508 ff.); Känner, KritV 2019, 57 (76). Skeptisch auch Morlok, ZRP 2017, 67 (68 f.) („Gefahr der Selbstverletzung der Demokratie“). Optimistischer Epping, Eine Alternative zum Parteiverbot, 2013, S. 80 f.; Ipsen, JZ 2017, 933 (935); Kluth, ZParl 2017, 676 (690); Morlok, NJW 2017, 3036 f.; Schwarz, NVwZ-Beil. 2017, 39 (41).

58 Vgl. Möllers, Demokratie - Zumutungen und Versprechen, 2009, Rn. 160.

59 Siehe zu dieser Erwägung auch Low, Trinity College Law Review 2018, 136 (151 f.).

60 Siehe https://www.verfassungsschutz.de/de/aktuelles/zur-sache/zs-2019-002-fachinformati on-zu-teilorganisationen-der-partei-alternative-fuer-deutschland-afd $<07 / 2020>$.

61 Siehe dazu § 11 Abs. 14 a AfD-Bundessatzung, 26.11.2015. 
tei, Gauland, hat den Flügel unmissverständlich der „Mitte der Partei“ zugerechnet. ${ }^{62}$ Auch führt die Auflösung des Parteiflügels nicht dazu, dass seine rechtsextremen Mitglieder aus der Partei ausgeschlossen würden. Konsequent wäre daher - rechtlich gesehen - die Beobachtung der Gesamtpartei, was aber - politisch betrachtet - hoch brisant ist, da die AfD sowohl auf Bundesebene als auch in einigen Landesparlamenten zur Oppositionsführerin geworden ist. Ein Verbot oder Finanzierungsausschluss der gesamten Partei ist jedenfalls nicht in Sicht.

\section{Justizreform als Einfallstor für autokratische Systemveränderun- gen}

In Anbetracht dieses ernüchternden Befundes stellt sich die Frage, ob sich die Resilienz des Grundgesetzes erhöhen lässt, um zumindest zu verhindern, dass unsere Demokratie von mehrheitsfähigen Populisten binnen kurzer Zeit zu einem autoritären Staat umgebaut wird. In der Türkei, Ungarn und Polen begannen die systemischen Umbrüche mit der Attacke mächtiger Vetoakteure wie obersten Gerichten, der freien Presse und Nichtregierungsorganisationen. ${ }^{63}$

\section{Aktuelle autoritär-populistische Angriffe auf die Judikative}

Die Justiz - in Deutschland insbesondere die Verfassungsgerichtsbarkeit - schützt in liberal-demokratischen Verfassungsordnungen Minderheitenrechte und politische Freiheiten von Oppositionsparteien. ${ }^{64}$ Vor der Folie ihres antagonistisch konzipierten Gesellschaftsbildes suchen autoritäre Populisten hingegen politische Gegner als Feinde des „wahren Volkes“ und Hindernis zur Durchsetzung des „wahren Volkswillens“ zu entrechten. Besonders in parlamentarischen oder semi-parlamentarischen Systemen - wie Polen, Ungarn und der Türkei - sehen sich Gerichte oft einem mehrdimensionalen Angriff durch autoritär-populistische Parteien ausgesetzt. ${ }^{65}$ Es gibt verschiedene Möglichkeiten, die Kontrolle über Gerichte zu erlangen: Durch die Besetzung der Gerichte mit Regierungsanhängern, die Reduzierung der Entscheidungskompeten-

62 Siehe https://www.tagesschau.de/inland/afd-verfassungsschutz-verdachtsfall-101.html $<07 / 2020>$.

63 Allgemein zu dieser Strategie von Populisten Arato (Fn. 13), S. 319 ff.; Mounk (Fn. 3), S. 9.

64 Für eine Beschränkung richterlicher Überprüfungsmacht im Hinblick auf Gesetze aber Waldron, Yale Law Journal 115 (2006), 1346 ff.

65 Siehe zur Situation in Ungarn European Commission for Democracy Through Law (Venice Commission), Opinion on Act CLI of 2011 on the Constitutional Court of Hungary, Opinion no. 665/2012, CDL-AD(2012)009; zur Situation in Polen European Commission for Democracy Through Law (Venice Commission), Poland Joint Urgent Opinion of the Venice Commission and the Directorate General of Human Rights and Rule of Law of the Council of Europe on Amendments the Law on the Common Courts, the Law on the Supreme Court, and Some Other Laws, Opinion No. 977/2019, CDL-Pl(2020)002; zur Situation in der Türkei European Commission for Democracy Through Law (Venice Commission), Opinion on the Law on the Establishment and Rules of Procedure of the Constitutional Court of Turkey, Opinion No. 612/2011, CDL-AD(2011)040. 
zen, die Änderung der Gerichtsorganisation (z.B. durch die Schaffung einer neuen Disziplinarkammer) oder die Änderung von Abstimmungsregeln. ${ }^{66}$

In Ungarn und der Türkei wurden die verfassungsrechtlichen Bestimmungen zur Ernennung der Verfassungsrichter verändert, um die richterliche Unabhängigkeit zu untergraben, und zugleich die Zuständigkeit des Verfassungsgerichts beschränkt. ${ }^{67}$ Die Partei „Recht und Gerechtigkeit“ (PiS) in Polen, die zunächst über keine verfassungsändernde Mehrheit verfügte, nahm einen Streit in der vergangenen Legislaturperiode über die Richterernennung zum Anlass, fünf neue Verfassungsrichter zu ernennen und änderte einfachgesetzlich zugleich die Mehrheitserfordernisse für die Ungültigkeitserklärung von Parlamentsgesetzen in ihren verschiedenen Reformen des Justizsystems. ${ }^{6}$ Später griff die PiS auch die Unabhängigkeit des Obersten Gerichtshofs - der höchsten Instanz der ordentlichen Gerichtsbarkeit - an, indem sie eine neue Disziplinarkammer schuf, die von der PiS durch ihre Mehrheit im Parlament besetzt wurde. ${ }^{69}$ Zurzeit tobt ein erbitterter Kampf der alten Richter gegen die neuen PiS-loyalen Richter des Obersten Gerichtshofs. Während die alten Richter - mit Unterstützung des $\mathrm{EuGH}^{70}$ - die neue Disziplinarkammer nicht als Teil der unabhängigen Justiz anerkennen, versucht die PiS mit einem neuen „Justizdisziplinargesetz“ neue Möglichkeiten zu schaffen, um sie loszuwerden. ${ }^{71}$

\section{Vulnerabilität der Deutschen Verfassungsgerichtsbarkeit}

Auch das BVerfG wäre verwundbar, wenn eine populistische Partei an die Macht käme. Zwar beschreibt Art. 93 GG die Kompetenzen des Verfassungsgerichts en détail und Art. 97 GG garantiert die richterliche Unabhängigkeit. Die verfassungsrechtlichen Bestimmungen des Art. 94 GG über die Ernennung von Richtern und die Organisation des Gerichts sind hingegen lückenhaft. ${ }^{72}$ Zur Richterwahl heißt es: „Das Bundesverfassungsgericht besteht aus Bundesrichtern und anderen Mitgliedern. Die Mitglieder des Bundesverfassungsgerichtes werden je zur Hälfte vom Bundestage und vom Bundesrate gewählt.” Im Hinblick auf die Gerichtsorganisation wird in Art. 94 Abs. 2 GG ganz auf das einfache Recht verwiesen - das BVerfGG.

66 Arato (Fn. 13), S. 321.

67 Zur Türkei Oder, Populism and the Turkish Constitutional Court, VerfBlog 2.5.2017, https: //verfassungsblog.de/populism-and-the-turkish-constitutional-court-the-game-broker-the-po pulist-and-the-popular/ $<07 / 2020>$.

68 Ausf. dazu Zoll/Worthham, Fordham International Law Journal 42 (2019), 875 (891ff.) Ein prägnanter Überblick findet sich auch bei Koncewicz, Constitutional Capture in Poland 2016 and Beyond, VerfBlog 19.12.2016, https://verfassungsblog.de/constitutional-capture-i n-poland-2016-and-beyond-what-is-next/ $<07 / 2020>$; Dworzynski, Und täglich grüßt das Murmeltier... die neue Justizreform der PiS in Polen, juwiss 88-2017, https://www.juwiss.d e $/ 88-2017 /<07 / 2020>$.

69 Zoll/Worthham, Fordham International Law Journal 42 (2019), 875 (894 ff.).

70 EuGH (A.K.), C-585/18, C-624/18 und C-625/18, EU:C:2019:982.

71 Siehe https://rsw.beck.de/aktuell/meldung/polen-gesetz-zur-richter-disziplinierung-passiertparlament $<07 / 2020>$. siehe auch Sanders, Muzzling Associations of Judges, VerfBlog 5.3.2020, https://verfassungsblog.de/muzzling-associations-of-judges/ $<07 / 2020>$.

72 So auch Walter, in: Maunz/Dürig (Hrsg.), GG, 88. EL 2019, Art. 94 Rn. 10. 
Nach $\S \S 6,7$ BVerfGG bedarf es für die Richterernennung einer Zwei-Drittel-Mehrheit. Außerdem schreibt $\S 6$ Abs. 2-4 BVerfGG vor, dass die vom Bundestag ernannten Richter nur auf Vorschlag eines repräsentativen Wahlausschusses, der aus $12 \mathrm{Ab}$ geordneten besteht, gewählt werden dürfen. Vorschläge des Wahlausschusses bedürfen der Zustimmung von drei Vierteln seiner Mitglieder. Das Verfahren gewährleistet somit ein Vetorecht der Oppositionsparteien und verhindert dadurch illegitimes ,court packing“" durch Regierungsfraktionen. ${ }^{73}$

Das BVerfGG selbst kann jedoch bereits durch einfache Mehrheit des Bundestages geändert werden. ${ }^{74}$ Die Zustimmung des Bundesrates ist nicht erforderlich; sein Einspruch kann gem. Art. 77 Abs. 3 und 4 GG leicht im Bundestag überkommen werden. Für eine populistische Partei, die bei Bundestagswahlen die absolute Mehrheit erreicht, wäre es daher ein Leichtes, im BVerfGG ein neues Ernennungssystem einzuführen, bei dem eine einfache Mehrheit im Bundestag genügt. ${ }^{75}$ Sie könnte die vom Bundestag zu bestimmenden Richter dann nach ihren eigenen Präferenzen ernennen. Um die Unparteilichkeit des Gerichts zu gefährden, reicht bereits die Einflussnahme auf nur die Hälfte der Richter eines Senats aus, da ein Verfassungsverstoß gem. $\S 15$ Abs. 4 BVerfGG nur dann festgestellt wird, wenn die Mehrheit der Richter hierfür votiert. Insbesondere im Hinblick auf die verfassungsrechtliche Überprüfung von Gesetzen ließe sich - etwa unter Berufung auf den international angesehenen Verfassungstheoretiker Waldron ${ }^{76}$ - auch ein qualifiziertes Mehrheitserfordernis einführen. Dies würde einer populistischen Partei erheblichen Spielraum für verfassungsrechtlich bedenkliche Praktiken verschaffen. Auch die Zuständigkeit der verschiedenen Senate ist nur durch das BVerfGG geregelt, so dass eine Partei - zusätzlich zu den neuen Ernennungsbestimmungen - mit absoluter Mehrheit auch einen neuen Senat schaffen könnte, dem alle staatsorganisationsrechtlichen Streitigkeiten zugewiesen werden. Steinbeiß hat kürzlich ein erschreckend realistisches fiktives Szenario hierzu entworfen. ${ }^{77}$

Man kann solche Veränderungen des BVerfGG mit Blick auf die in Art. 97 GG verbriefte richterliche Unabhängigkeit als verfassungswidrig ansehen. ${ }^{78} \mathrm{Ob}$ sich das Gericht jedoch in der Verfassungsrealität solcher legislativen Angriffe erwehren könnte, ist - insbesondere vor dem Hintergrund der Entwicklungen in Polen - äußerst frag-

73 Vgl. Möllers, in Jestaedt/Lepsius/Möllers/Schönberger (Hrsg.), Das entgrenzte Gericht, 2011, 281 (360).

$74 \mathrm{Zu}$ dieser Gefahr auch schon Steinbei $\beta$, Das Grundgesetz in Zeiten von AfD \& Co., Tagesspiegel, 22.5.2019, https://www.tagesspiegel.de/politik/70-jahre-grundgesetz-das-grundges etz-in-zeiten-von-afd-und-co-/24357768-2.html <07/2020>.

75 Das würde für sich genommen auch keinen Verfassungsbruch darstellen. Auch in vielen Bundesländern genügt die einfache Mehrheit; siehe etwa Art. 68 Abs. 3 S. 2 Landesverf. BW; Art. 68 Abs. 2 Landesverf. BY; Art. 112 Abs. 4 S. 1 Landesverf. BB; Art. 65 Abs. 2 S. 1 Landesverf. $\mathrm{HH}$.

76 Waldron, Yale Law Journal 115 (2006), $1346 \mathrm{ff}$.

77 Steinbeis, Ein Volkskanzler, VerfBlog 9.9.2019, https://verfassungsblog.de/ein-volkskanzler $/<07 / 2020>$.

78 Siehe zu den Grenzen legislativer Einflussnahme auch Hillgruber, in: Maunz/Dürig (Hrsg.), GG-Kommentar, 88. EL 2019, Art. 97 Rn. 87 ff. 
lich. ${ }^{79}$ Eine Verfassung enthält immer normative Lücken, um offen für demokratische Veränderungen zu sein. Das BVerfG stellt jedoch sicher, dass die Offenheit des Verfassungstextes nicht für eine Monopolisierung der Macht im Namen der Volkssouveränität missbraucht wird. ${ }^{80} \mathrm{Im}$ deutschen Rechtskontext nimmt die Verfassungsgerichtsbarkeit eine Schlüsselrolle für die Erhaltung der liberalen Demokratie ein. ${ }^{81}$ Fällt dieses institutionelle Bollwerk, so öffnet sich autoritären Populisten Tür und Tor, um Wahlgesetze zu ihren Gunsten zu verändern, öffentlich-rechtliche Rundfunkanstalten unter ihre Kontrolle zu bringen, ${ }^{82}$ die freie Presse zu untergraben und politische wie gesellschaftliche Oppositionsbewegungen zu zerschlagen.

\section{Vorschläge zur Stärkung der Widerstandsfähigkeit des deutschen Grundgesetzes}

Man kann natürlich meinen, dass die Suche nach konstitutionellen Schutzmaßnahmen sinnlos sei, da ein Staatssystem, das die Mehrheit der Bevölkerung ablehnt, kaum rechtlich gesichert werden könne. Allerdings repräsentiert die absolute Mehrheit im Parlament nicht notwendiger Weise die tatsächliche Mehrheit der Wählerschaft. In Mehrparteiensystemen mit prozentualer Hürde reichen - je nach politischer Konstellation - weit weniger Stimmen aus. In Polen etwa erreichte die PiS-Partei im Jahr 2015 bereits mit einem Anteil von nur 37,6 \% Stimmen eine absolute Mehrheit im Parlament. Es lohnt sich daher, die Resilienz der Verfassung zu gewährleisten, um die Wettbewerbsdemokratie zu schützen. Dazu bedarf es einer besseren verfassungsrechtlichen Absicherung der Unabhängigkeit der Justiz. ${ }^{83}$

Um die Autonomie des Bundesverfassungsgerichts zu sichern, kommen verschiedene Maßnahmen in Betracht: Zum einen könnte die qualifizierte Mehrheit für die Wahl von Verfassungsrichtern, die derzeit in $\S 6$ Abs. 1 und $\S 7$ BVerfGG vorgesehen ist, in Art. 94 Abs. 1 GG verankert werden. ${ }^{84}$ Ein weiterer entscheidender Faktor für die Gewährleistung der Unabhängigkeit der Richter ist ihre Amtsdauer. Sie sollte mindestens doppelt so lang wie die Legislaturperiode sein, um die richterliche Unabhängig-

$79 \mathrm{Zu}$ dieser Gefahr auch Steinbeis, Das Grundgesetz in Zeiten von AfD \& Co., Tagesspiegel, 22.5.2019, https://www.tagesspiegel.de/politik/70-jahre-grundgesetz-das-grundgesetz-in-ze iten-von-afd-und-co-/24357768-2.html <07/2020>. Optimistischer hingegen Müller, Jeder, der versuchen würde, das Bundesverfassungsgericht auszuhebeln, würde sich verheben, VerfBlog, 24.2.2017, https://verfassungsblog.de/jeder-der-versuchen-wuerde-das-bundesve rfassungsgericht-auszuhebeln-wuerde-sich-verheben/ $<07 / 2020>$.

80 Allgemein zur Rolle der Justiz im Populismus-Kontext Arato (Fn. 13), S. 331.

81 Siehe zur besonderen Rolle des BVerfG auch in rechtsvergleichender Hinsicht Jestaedt, in: Das entgrenzte Gericht (Fn. 73), S. $77 \mathrm{ff}$.

82 Siehe dazu im polnischen Kontext Berka/Tretter, MR-Int 2016, $118 \mathrm{ff}$.

83 Hierfür hatte sich zu Recht auch Justizsenator Till Steffen der Freien und Hansestadt Hamburg im Zuge der Herbstkonferenz der Justizministerinnen und Justizminister am 7.11.2019 in Berlin eingesetzt.

84 Hierfür auch Gärditz, Die meisten Dinge, die in Polen und Ungarn gelaufen sind, könnten ohne weiteres hier auch passieren, VerfBlog 22.2.2018, https://verfassungsblog.de/die-meis ten-dinge-die-in-polen-und-ungarn-gelaufen-sind-koennten-ohne-weiteres-hier-auch-passie ren/ <03/2020>; Karpenstein, NJW-aktuell 48/2019, Editorial. 
keit zu gewährleisten. ${ }^{85}$ Eine solche Garantie könnte in Art. 94 GG oder Art. 97 GG aufgenommen werden. ${ }^{86}$

Schließlich zeigt das polnische Beispiel, dass auch die Regeln der Gerichtsorganisation anfällig für missbräuchliche Gesetzesänderungen durch autoritär-populistische Akteure sind. Eine Möglichkeit, die Organisationsstrukturen des Bundesverfassungsgerichts gegen undemokratische Einflüsse abzusichern und gleichzeitig die Flexibilität, die das einfache Recht im Vergleich zum Verfassungsrecht bietet, zu erhalten, könnte darin bestehen, dem Bundesrat bei der Änderung des BVerfGG eine Vetoposition einzuräumen. Dazu müsste man lediglich Art. 94 Abs. 2 GG um den Satz ergänzen: „Das Gesetz bedarf der Zustimmung des Bundesrates““. 87

\section{E. Fazit}

Zusammenfassend lässt sich feststellen, dass die Grundannahmen des autoritären Populismus verfassungsrechtlichen Grundprinzipien liberaler Demokratien in mehrfacher Hinsicht widersprechen. Dennoch lassen sich autoritär-populistische Parteien in der Verfassungsrealität weder verbieten noch anderweitig rechtlich sanktionieren, da die Wettbewerbsdemokratie von der Neutralität gegenüber politischen Ansichten und der Offenheit des politischen Diskurses lebt. Zumindest aber können moderate Grundgesetzänderungen zur Sicherung der Verfassungsgerichtsbarkeit vorgenommen werden, um zu verhindern, dass Populisten mit einer absoluten parlamentarischen Mehrheit die Demokratie allzu leicht in eine Autokratie verwandeln. Letztlich wird damit nur das Symptom, nicht aber die Ursache des autoritären Populismus bekämpft. Dazu bedarf es gesamtgesellschaftlicher Anstrengungen, um die liberale Demokratie wieder für alle Teile des Volkes attraktiv zu machen.

85 Siehe dazu - auch in verfassungsvergleichender Hinsicht - Hönnige, ZSE 3/2008, 524 (539).

86 Auf landesverfassungsrechtlicher Ebene existieren solche Bestimmungen bereits. Siehe etwa Art. 76 Abs. 2 S. 1 Landesverf. NW: „Die Mitglieder und ihre Stellvertreter werden vom Landtag ohne Aussprache mit Zweidrittelmehrheit auf die Dauer von zehn Jahren gewählt.“ Vgl. auch Art. 79 Abs. 3 Landesverf. TH; Art. 81 Abs. 3 Landesverf. SN.

87 Hierfür auch Gärditz, Die meisten Dinge, die in Polen und Ungarn gelaufen sind, könnten ohne weiteres hier auch passieren", VerfBlog 22.2.2018, https://verfassungsblog.de/die-mei sten-dinge-die-in-polen-und-ungarn-gelaufen-sind-koennten-ohne-weiteres-hier-auch-passi $\mathrm{eren} /<07 / 2020>$. 